\title{
RursuSpicae
}

Transmission, réception et réécriture de textes, de I'Antiquité au Moyen Âge

\section{Le phénix dans le Physiologus byzantin du pseudo- Epiphane et dans le Physiologus de Vienne : erreur textuelle et interprétation étymologique}

The Phoenix in the Byzantine Physiologus by Pseudo-Epiphanius and in the Vienna Physiologus : a Textual Mistake and an Etymological Interpretation

\section{Françoise Lecocq}

\section{CpenEdition}

Journals

\section{Édition électronique}

URL : http://journals.openedition.org/rursuspicae/818

DOI : $10.4000 /$ rursuspicae. 818

ISSN : 2557-8839

\section{Éditeur :}

Université Nice-Sophia Antipolis, IRHT - Institut de recherche et d'histoire des textes

\section{Référence électronique}

Françoise Lecocq, «Le phénix dans le Physiologus byzantin du pseudo-Epiphane et dans le

Physiologus de Vienne : erreur textuelle et interprétation étymologique », RursuSpicae [En ligne], 2 I

2019, mis en ligne le 20 décembre 2019, consulté le 29 mars 2021. URL : http://

journals.openedition.org/rursuspicae/818; DOI : https://doi.org/10.4000/rursuspicae.818

Ce document a été généré automatiquement le 29 mars 2021.

RursuSpicae 


\section{Le phénix dans le Physiologus byzantin du pseudo-Epiphane et dans le Physiologus de Vienne : erreur textuelle et interprétation étymologique}

The Phoenix in the Byzantine Physiologus by Pseudo-Epiphanius and in the Vienna Physiologus : a Textual Mistake and an Etymological Interpretation

Françoise Lecocq

\section{Introduction}

1 Hérodote raconte, dans sa notice sur la faune (et non la religion) de l'Égypte, que le jeune phénix transporte depuis l'Arabie le corps de son père dans un œuf de myrrhe afin de lui rendre les honneurs funèbres au temple d'Héliopolis (HÉRODOTE 2.73). Cette boule d'aromates qui renferme les restes paternels, en une sorte de momification inversée, est paradoxalement un œuf de mort et non un œuf de vie, car le phénix ne pond pas : il renait de la putréfaction du cadavre, puis, dans une version plus tardive du mythe, de ses cendres. C'est dans ces termes que l'historien grec présente le benu, l'oiseau solaire sacré attesté par nombre de textes et d'images dans la civilisation égyptienne. Dans la cosmogonie héliopolitaine, il est la première créature, à l'origine du monde, à se poser sur la terre émergée des eaux. Il symbolise au premier chef le cycle quotidien du soleil levé à l'orient, couché à l'occident et traversant le monde souterrain en barque pendant la nuit pour reprendre sa place. Dans les représentations, sur les peintures tombales ou sur les vignettes des papyrus du Livre des morts, il transporte fréquemment le soleil dont il est une figure, mais posé sur sa tête, un globe rouge qu'on voit également pour le faucon solaire Horus avec lequel il peut se confondre, comme le disent les formules des prières. Jamais il n'est montré 
transportant l'astre dans ses pattes, ni en plein vol, mais il porte parfois sur la tête l'attribut royal de la couronne pharaonique, à la place du globe solaire (fig. 1, 2 et 3).

2 Quand l'oiseau, rare dans la littérature grecque, est adopté et adapté à Rome, il garde le nom de phoinix que lui a donné, sans l'expliquer, Hérodote. Le disque solaire sur la tête des images égyptiennes et l'œuf aux pattes du récit hérodotéen, en principe exclusifs l'un de l'autre puisque tous deux sont des images du soleil, subissent des métamorphoses iconographiques et symboliques, et vont jusqu'à être s'associés ${ }^{1}$. Le premier se transforme en une couronne radiée, sur un modèle hellénistique d'attribut du pouvoir, avec une reconnaissance officielle de l'oiseau tentée par l'empereur Claude $^{2}$, puis réalisée par Hadrien, sur des monnaies en l'honneur de son père adoptif Trajan: le nouveau symbole exprime principalement la transmission dynastique du pouvoir et le renouveau heureux des temps ${ }^{3}$ (fig. 4 et 5). Quant à la boule de myrrhe funéraire, dont on parle toujours aux 1er-2ème siècles (TACITE, Ann. 6.28), mais qui disparait ensuite souvent des textes au profit de la variante de la mort et de la renaissance dans les flammes, elle devient durablement dans la numismatique impériale (également dès Hadrien), un globe, qui n'est pas l'œuf d'Hérodote, ni même l'astre solaire ${ }^{4}$, mais plutôt l'orbe terrestre ou cosmique, dans une typologie semblable à celle de l'aigle jupitérien et de la victoire ailée portés dans la main d'un personnage. Sa signification est de manifester, selon les cas, l'éternité individuelle d'un empereur ou de son épouse (fig. 6), ou bien celle du monde cycliquement régénéré ou bien la domination romaine sur l'ensemble de l'orbis terrarum.

Vers la fin du 1er siècle de notre ère, le christianisme adopte à son tour le phénix, tout d'abord comme exemple naturel de la résurrection des corps (CLÉMENT de Rome, Ép. 1.25). Dans les siècles suivants, le bestiaire chrétien populaire qu'est le Physiologus interprète symboliquement les caractéristiques des différents animaux pour y voir des vertus et des messages analogiques de ceux du Christ; notre oiseau y figure en bonne place ${ }^{5}$. L'œuvre a été traduite en plusieurs langues, à différentes époques et s'est aussi accrue de nombreuses variantes; celles-ci peuvent renvoyer à des sources précises, mais elles peuvent également être soit des inventions, soit des erreurs de diverses natures ${ }^{6}$. Nous nous proposons de rendre compte de deux de ces variantes concernant l'oiseau unique, dont l'une s'explique par la mélecture d'un mot et l'autre est une explication étymologique. Nous étudierons d'abord le phénix, la coquille et le marteau dans le Physiologus byzantin du pseudo-Épiphane, puis nous traiterons d'une étymologie de poĩvı proposée par le Physiologus de Vienne, peut-être pas entièrement nouvelle : « celui qui apparaît ».

\section{Le phénix, la coquille et le marteau dans le Physiologus byzantin du pseudo-Epiphane ${ }^{7}$}

4 Si toutes les versions du Physiologus comportent une notice sur le phénix, toutes n'en donnent pas une description. La plupart vont droit à l'essentiel: le récit de sa résurrection et son symbolisme chrétien. Celle du Physiologus byzantin attribué au pseudo-Épiphane, datée entre le $5 \mathrm{e}$ et le $11 \mathrm{e}$ siècle, fait un portrait de l'oiseau, comportant plusieurs différences par rapport au standard du mythe. Le texte commence par une comparaison avec le plumage du paon (au désavantage de celui-ci),

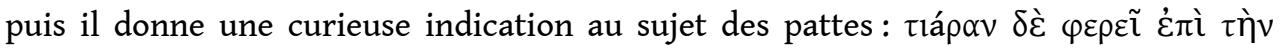

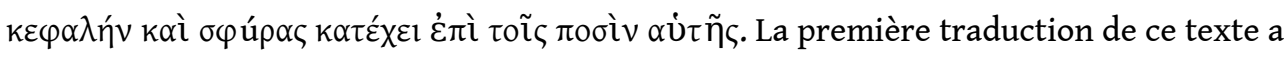


été latine, et l'édition de la Patrologie grecque de Migne a repris l'interprétation de Gonzalo Ponce de Leon : coronam autem habet in capite, et in pedibus malleolos ${ }^{8}$, c'est à dire «il a une couronne ${ }^{9}$ sur la tête et des malléoles aux pattes». Le savant espagnol ne commente pas ce détail, et l'illustration de l'édition anversoise montre des pattes d'oiseaux normales ${ }^{10}$.

D'où viennent ces étranges «malléoles»? Malleolus est le diminutif de malleus, qui désigne un «marteau » et aussi une arme de guerre : un «trait enflammé ». Aucun de ces deux objets ne paraît pertinent pour le phénix. Le second sens explique pourtant la glose d'un commentateur anglophone contemporain à partir de la traduction latine: It has a crested head and feet like fire ${ }^{11}$. Les pattes ressembleraient ici à du feu - ce que ne dit aucune autre source ${ }^{12}$; quand les auteurs décrivent les pattes, c'est pour leur couleur

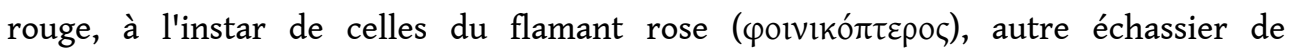
l'Égypte ${ }^{13}$. De plus, malleolus n'a pas en latin classique le sens moderne d'os de la cheville, et les oiseaux n'ont pas ces excroissances du tibia et du péroné.

6 Il est vrai que dans ce Physiologus, il y a un développement sur les pattes du paon, l'animal qui fait suite au phénix ; mais ce développement est signifiant, expliquant que l'oiseau se met à crier quand il voit qu'elles n'ont pas la beauté du reste de son corps. Pour le phénix, on ne comprend guère l'intérêt de cette précision. Les autres versions du Physiologus qui mentionnent les pattes de ce dernier disent qu'il transporte avec elles, comme avec ses ailes, les aromates de son bûcher ${ }^{14}$, par exemple chez le pseudo-

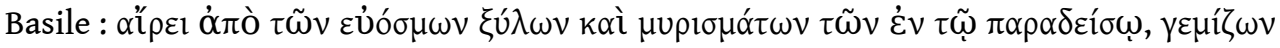

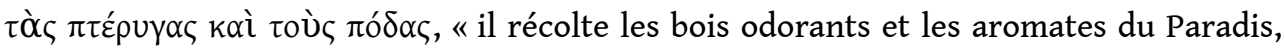
en charge ses ailes et ses pattes $»^{15}$. Olaus Gerhardus Tychsen citait ce passage dans son édition du Physiologus syriaque, où il donnait aussi la traduction latine de Ponce de Leon; mais il semble en avoir subodoré la bizarrerie en mettant le mot grec $\sigma \varphi u ́ p \alpha \varsigma$ entre parenthèses à côté de malleolos ${ }^{16}$.

7 Les spécialistes contemporains du mythe du phénix ont, eux, traduit ou commenté ce texte du pseudo-Épiphane, sans résoudre l'énigme des "malléoles », et sans peut-être même la voir. Jean Hubaux et Maxime Leroy, puis Roelof van den Broek rapprochent le passage de l'œuvre de Saint Épiphane où sont mentionnés «les tarses» de l'oiseau, dont il se bat le corps pour provoquer les étincelles qui allumeront son bûcher : $\tau \alpha \rho \sigma o i \varsigma \varsigma$

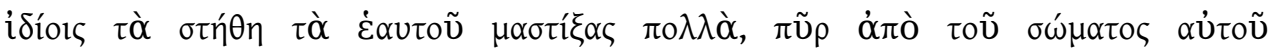

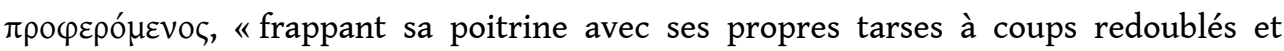
mettant le feu à son corps " (ÉPIPHANE, Ancor. 84) ${ }^{17}$. Tarses des pattes ou des ailes? se demande le savant néerlandais, puisque le mot a ces deux emplois. La traduction latine par Iohannes Gerhard de cette phrase était une glose qui ne choisissait pas entre l'un ou l'autre sens, $\tau \alpha \rho \sigma o ́ c$ étant rendu à la fois par «pattes» et par «ailes»: propriis pedibus ac alis pectora sua ualde pulsans ignemque a corpore suo proferens, "frappant vigoureusement sa poitrine avec ses propres pattes et ailes et mettant le feu à son corps $\aleph^{18}$. Mais $\tau \alpha \rho \sigma o ́ \varsigma$ désigne une surface plane, donc la plante des pieds (et non les chevilles), comme a choisi de traduire Francesco Sbordone : E dopo d'essersi piu volte percosso il petto con le piante dei piedi ${ }^{19}$; cependant, les oiseaux n'ayant pas de plante des pieds, le mot désigne en fait leurs serres, par exemple chez les poètes ${ }^{20}$. Pour le PseudoÉpiphane, Hubaux et Leroy ont traduit, plus vraisemblablement, par « ailes » (HuBAUx, LEROY, 1939 : 158), ce qui est plus naturel et plus pratique à battre que les pattes. 
En fait, les «marteaux - malléoles » du phénix n'existent tout simplement pas : il ne s'agit que d'une coquille. Ponce de Léon a choisi malleolus pour traduire $\sigma \varphi \tilde{v} \rho \alpha$ " marteau », qui n'a pas de sens anatomique en grec, pas plus que malleolus en latin; il a

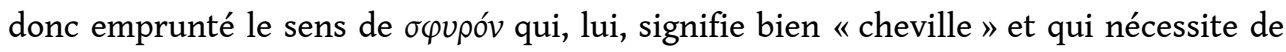

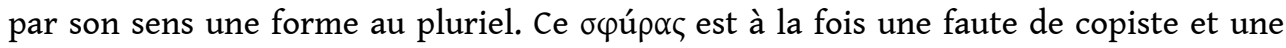
hypercorrection à partir d'une mélecture de $\sigma \varphi \alpha \tilde{i} \rho \alpha v$ « la sphère » (au singulier), en l'occurrence le globe sur lequel le phénix est souvent perché dans la numismatique romaine, soit comme sujet principal de l'image, soit comme attribut porté ou offert par un personnage, avec des valeurs symboliques diverses selon la légende monétaire et le contexte historique. Couronne radiée et orbe parfois fascié sont les attributs officiels du phénix sur les documents impériaux, des monnaies des Antonins à celles des Constantiniens et au-delà ${ }^{21}$ (fig. 7). La source de ce Physiologus du Pseudo-Épiphane décrivait simplement l'image classique de l'oiseau sous l'empire romain, exactement comme dans la version byzantine du Physiologus, qui explicite le sens de ces attributs

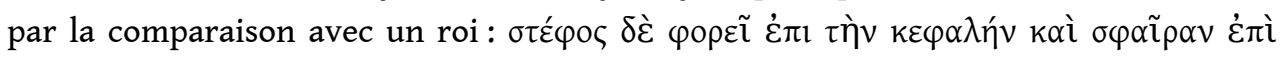

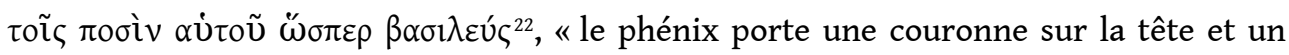
globe à ses pieds, comme un roi » (fig. 8). Ce dernier texte n'est cependant pas non plus exempt de bizarrerie, puisqu'il dit plus loin que l'oiseau «allume le feu avec ses

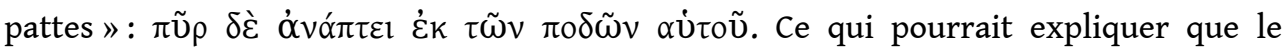
commentateur anglophone que nous avons mentionné n'ait pas trouvé si étrange que les pattes de l'oiseau aient l'apparence du feu (feet like fire).

Le manuscrit Roma, Bibl. Vallicelliana, F 68 nous livre le chaînon manquant, avec un texte d'un état intermédiaire entre cette dernière version byzantine, sans faute, et celle, fautive, du Pseudo-Épiphane : la diphtongue de $\sigma \varphi \alpha i ̃ \rho \alpha v$ y est déjà remplacée par

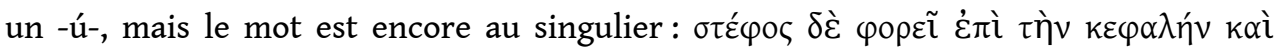

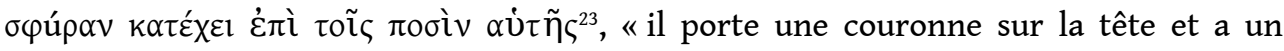
marteau [pour "globe"] à ses pieds ».

Pour conclure sur un plan méthodologique, nous remarquons une fois de plus la tendance à prendre les textes sur le phénix au premier degré et à les considérer comme entièrement fiables; mais les nombreux détails et variantes du mythe sont loin d'être tous des informations authentiques remontant à quelque source perdue, comme on le lit trop souvent. Ce peuvent être soit des emprunts, soit de pures inventions - et pas seulement dans le cas des poètes -, soit encore des confusions, comme par exemple plusieurs fois chez Pline l'Ancien, nous l'avons démontrée ${ }^{24}$. Ici, l'étrangeté du détail sur les pattes s'explique simplement par une erreur matérielle, une coquille de copiste.

\section{Une nouvelle étymologie de $\varphi$ oĩvı $\xi$ dans le Physiologus de Vienne : "celui qui apparaît »}

11 La notice sur le phénix la plus surprenante et la plus atypique de toutes les versions $\mathrm{du}$ Physiologus est celle de Vienne, appartenant à la famille de la première recension. Il ne s'agit pas de la première notice, qui classe le phénix vers le début de sa liste, en

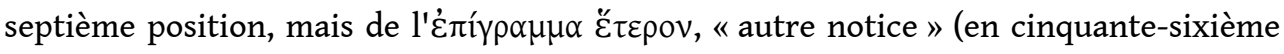
position) qui se situe à la fin et qui comporte dans ses premières lignes descriptives plusieurs détails inédits. 


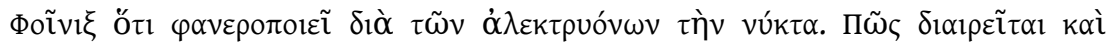

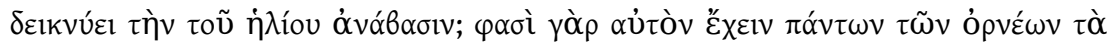

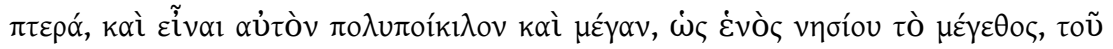

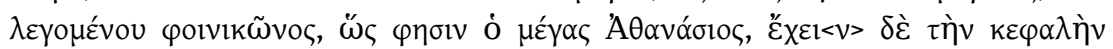

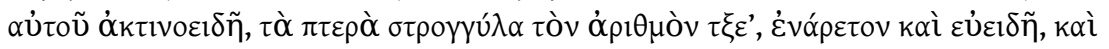

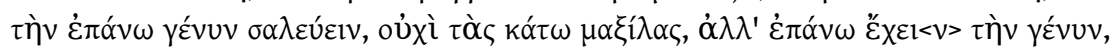
ò

Phénix, parce qu'il fait la nuit claire par l'intermédiaire des coqs. Comment séparet-il (la nuit du jour) et comment indique-t-il le lever du soleil ? On dit qu'il a les plumes de tous les oiseaux et qu'il est très bigarré et de haute taille, aussi grand que l'îlot appelé Phoinikôn, comme le dit le grand Athanase. Sa tête est ornée de rayons ; ses plumes, qui sont arrondies, sont au nombre de 365. Il est magnifique et beau à voir. C'est sa mandibule supérieure qui se meut et non celle d'en dessous : il ne soulève que la partie supérieure de son bec. Il a les pieds faits d'un seul morceau de chair et membranés comme ceux des oies et des canards $[. . .]^{25}$.

Cette description emprunte au congénère égyptien de l'oiseau, le crocodile, sa mandibule supérieure mobile (HÉRODOTE 2.68.3) : c'est une sorte de contamination par voisinage ${ }^{26}$. Elle comporte aussi une indication géographique mystérieuse: l'île Phoinikôn, c'est à dire peut-être une palmeraie insulaire ; cette version du Physiologus serait ainsi la seule à mentionner au sujet de l'oiseau l'arbre homonyme, qui lui est fréquemment associé dans les textes et surtout l'iconographie chrétienne ${ }^{27}$. Outre le problème de sa localisation, l'île constitue bizarrement la mesure de la taille gigantesque du phénix, avec la caution non moins curieuse du "grand Athanase». Il s'agirait du fameux évêque d'Alexandrie adversaire de l'hérésie arienne sous Constantin ${ }^{28}$, mais ses œuvres conservées ne parlent pas de l'oiseau ${ }^{29}$. De manière plus évidente, les plumes qui auréolent la tête peuvent être imaginées comme une sorte de roue comparable à celle qui encercle la tête du paon montré de face, tel qu'il apparaît sur nombre de monnaies et de mosaïques; pour le phénix, l'important est le chiffre astronomique (aux sens propre et figuré du terme) de 365, qui est le nombre des jours de l'année solaire et qui fait de lui une créature colossale, d'une échelle cosmique.

\subsection{Un oiseau héliodrome}

Ce gigantisme et ce chiffre nous donnent la clé du texte : il s'agit d'un emprunt aux religions orientales où un oiseau géant accompagne l'astre du jour dans sa course quotidienne, remplissant le rôle d'héliodrome («coursier du soleil»). Il est perçu comme tantôt bénéfique, tantôt maléfique : il protège la terre des ardeurs excessives de ses rayons, ou bien il fait obstacle à ces mêmes rayons dispensateurs de vie. Parfois un griffon, souvent un coq, il peut servir de monture à la divinité solaire. Citons le gigantesque oiseau rebelle Anzu chez les Sumériens ${ }^{30}$, le coq sacré perse Parodarsh ${ }^{31}$ et l'oiseau Garuda compagnon de Vishnu ${ }^{32}$. Les légendes rabbiniques le connaissent comme un être malfaisant sous le nom de Ziz: l'envergure de ses ailes cache le soleil à la terre ; il règne sur les airs comme Léviathan sur les eaux et Béhémot sur les terres ${ }^{33}$; de même que les deux autres monstres, il sera mangé au banquet des justes à la fin des temps. Il est vu tel un griffon ${ }^{34}$ ou un oiseau aux pieds palmés, et un manuscrit illustré du Talmud babylonien en montre une image, sous le nom de Bar Iokni : c'est une sorte de canard bleu et rouge ${ }^{35}$ (fig. 9). Et de fait, le Physiologus de Vienne attribue des pattes palmées à son "phénix ". Mais peut-être est-ce simplement aussi, comme pour la mandibule, une contamination par voisinage avec un autre animal : un roman grec par 
exemple décrit le griffon indien aux pattes palmées dans le paragraphe précédant celui sur le phénix (Philostrate, V. Apoll. 3.48 -49).

\subsection{Une nouvelle étymologie : l'oiseau « qui apparaît »} apparait dès le titre de la notice : il s'agit d'une nouvelle étymologie de poĩvı rien à voir avec les explications fournies précédemment ou ultérieurement par d'autres auteurs, comme l'homonymie avec le palmier où il se perche sur les mosaïques des églises (fig. 10) autant que dans les textes (LACTANCE, De aue phoen.69-70; ISIDORE, Étym. $17.7^{36}$ ) ou avec le pays des Phéniciens où il séjourne (également Lactance ${ }^{37}$ ), avec la couleur de la teinture pourpre - d'ailleurs originaire de Phénicie - de son plumage (ISIDORE, Étym.12.7), ou encore avec un prétendu mot arabe signifiant « unique, célibataire » (encore ISIDORE, Étym. 12.7). Elle n'a rien à voir non plus, bien sûr, avec les théories modernes, selon lesquelles $\varphi$ oĩv $\xi$ signifierait originellement en grec « rouge sang », d'où « brillant $»^{38}$. retenu l'attention des éditeurs et commentateurs, alors que les remarques philologiques sont une spécificité de cette version viennoise du Physiologus : le savant italien lui-même la balaie d'un revers de mots pour son «absurdité » (SBORDONE, 1935 : 29). Sans doute, elle est fausse du point de vue de la science contemporaine, mais, loin d'être absurde, elle est signifiante et intéressante à un autre titre que linguistique, bien plus que la proposition faite par la première notice sur le phénix dans le même manuscrit de ce Physiologus qui en comporte deux, comme nous l'avons dit:

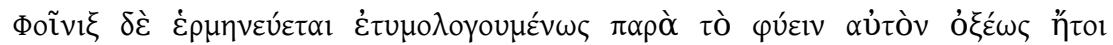

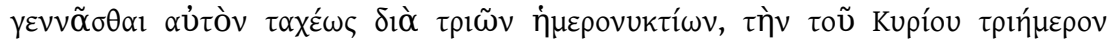

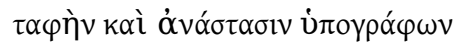

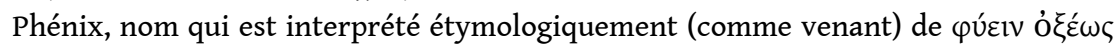
('engendrer rapidement'), parce que cet oiseau se produit lui-même en peu de temps, (savoir) en trois jours et trois nuits, figurant l'ensevelissement du Seigneur et sa résurrection après trois jours. ${ }^{39}$

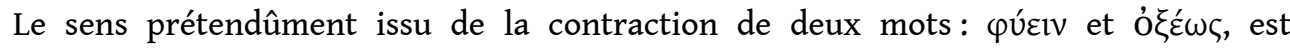
évidemment à mettre en rapport moins avec les racines grecques qu'avec la croyance chrétienne de la résurrection du Christ en trois jours, une durée que les Physiologus transfèrent à la renaissance de l'oiseau (et ce, bien sûr, à la date de Pâques) ${ }^{40}$.

Dans la seconde notice, la créature gigantesque est un coq solaire, chef des coqs terrestres ${ }^{41}$; elle est dite chanter au lever du soleil, les deux " apparaissant » en même temps. Elle porte donc précisément le nom de «phénix » en tant qu'oiseau qui «fait

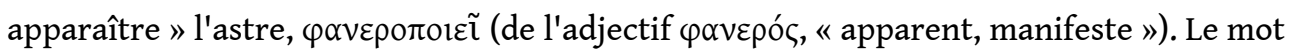

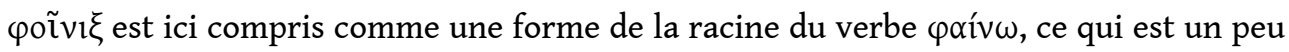
plus linguistiquement possible et plus sémantiquement plausible que le rapprochement de la notice précédente.

\subsection{Une étymologie peut-être pas si neuve}

Or, nous pensons voir peut-être aussi cette même étymologie, d'une manière implicite, chez Eusèbe de Césarée quand il introduit la citation du passage sur le mystérieux 
oiseau de L'Exode d'Ézéchiel le Tragique, un Juif alexandrin hellénophone du 2e s. avant notre ère. Il le fait à la suite de la description d'Ézéchiel qui comporte elle-même, trois fois en huit lignes, une forme de ce verbe paív $\omega$, toujours placée en fin de vers : plutôt qu'une grande maladresse stylistique, il pourrait s'agir de clins d'œil étymologiques pour faire reconnaître la créature, qui est ici aussi le roi de la basse-cour au cri sonore, ennobli du nom de " phénix ». De plus, l'oasis où il se trouve s'est révélée aux éclaireurs

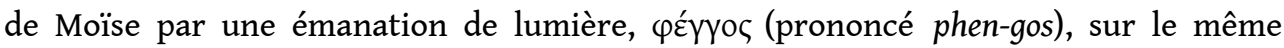
radical que $\varphi \alpha i ́ v \omega$ :

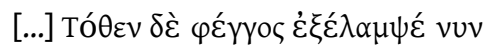

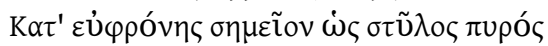

[...] [Ce lieu] se trouve là-bas, d'où un éclat a brillé pendant la nuit, signe pareil à une colonne de feu. ${ }^{42}$

19 Ces indices sur l'identité de l'animal viennent renforcer celui de sa présence dans un

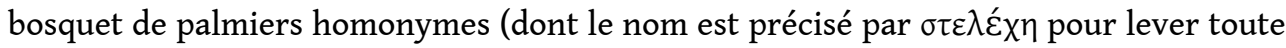
ambiguïté43) : l'oiseau "de lumière " " apparaît », et il apparaît dans les "palmiers » homonymes. Eusèbe présente l'extrait et recopie ensuite la description de la créature :

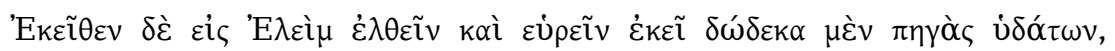

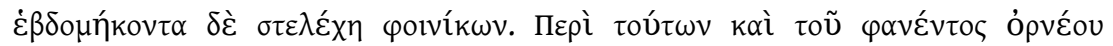

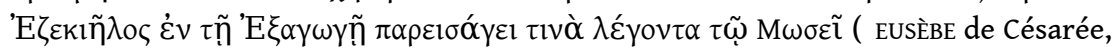
PE 9.29)

De là ils vinrent à élim, où ils trouvèrent douze fontaines et soixante-dix palmiers. Ézéchiel fait ici intervenir un interlocuteur, dans son 'E $\xi \alpha \gamma \omega \gamma \eta$; lequel adressant la parole à Moïse, lui parle de ces choses et d'un oiseau qu'il a vu [ou, plus exactement selon nous : « qui apparait » $]^{44}$.

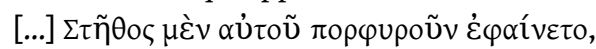

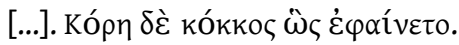

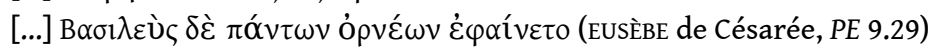

[...] Sa poitrine apparaissait de couleur pourpre [...]. Et la pupille apparaissait comme un pépin. Il avait le chant le plus remarquable de tous. Il apparaissait comme le roi de tous les oiseaux ${ }^{45}$.

Dans ce fragment, l'animal n'est pas nommé, parce que l'œuvre n'est citée que partiellement et/ou plutôt parce que cet oiseau rare est inconnu des personnages de la pièce. Et si ce n'était pas un phénix chez Ézéchiel (mais quel autre oiseau merveilleux pourrait-ce être si le jeu étymologique est avéré ? ${ }^{46}$, en tout cas le pseudo-Eustathe, qui cite le même passage qu'Eusèbe ${ }^{47}$, le baptise expressément de ce nom en des temps où il faisait parfaitement sens, car il était devenu un symbole universel, à la fois présage impérial et miracle chrétien. Cependant, Ézéchiel le décrit bien comme un coq, avec la caractéristique d'une pupille jaune, ajoutée à celle de son chant, indépendamment de l'absence d'une queue remarquable et du problème textuel de la lecture et du sens du

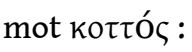

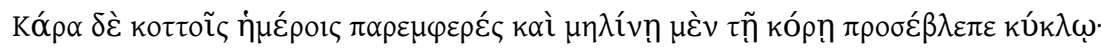

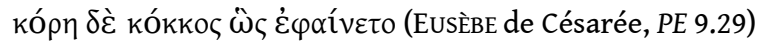

Quant à la tête, il était presque semblable aux coqs domestiques et il regardait tout autour avec une prunelle de couleur jaune coing. Et la prunelle apparaissait comme un pépin ${ }^{48}$.

21 Et c'est encore à la lumière du même jeu de mots sur $\varphi \alpha i ́ v \omega$ qu'on pourrait comprendre la curieuse indication du roman Leucippé et Clitophon, au moment où le phénix se fait

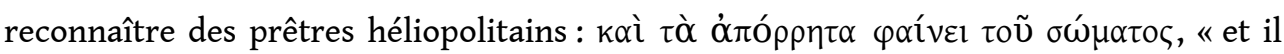
fait apparaître les secrets de son corps » (ACHILLE TATIUS, Leuc. et Clit. 3.25); ce serait un 
jeu étymologique plutôt qu'un euphémisme pour désigner ses parties sexuelles, comme cela a été proposé - si tant est que le phénix soit pourvu d'un sexe ${ }^{49}$. Il se révèle bien en effet comme l'oiseau " qui apparaît ", c'est à dire le poĩvił. Il se trouve également que, dans son passage sur la création des oiseaux, le ps. Eustathe cite pêle-mêle au sujet du phénix non seulement L'Exode d'Ézéchiel le Tragique, mais justement aussi Leucippé et Clitophon, ainsi que le Physiologus. Les différentes graphies de "phénix » en grec et en latin à travers le temps attestent des diverses prononciations du mot ${ }^{50}$.

Ainsi, en remontant encore plus haut dans le temps, nous remarquons que figure déjà chez Aristophane un double jeu de mots étymologique sur le nom du phénix, ici à des fins humoristiques. Dans les Acharniens, l'ambassadeur grec se voit servir à la table du roi de Perse des mets extraordinaires : un bœuf entier rôti au four et un oiseau géant $\varphi \varepsilon ́ v \propto \xi$ qui fait trois fois la taille d'un homme (ARISTOPHANE, Acharn. 88-99). Serait-ce une

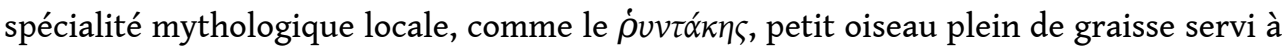
la table d'Artaxerxès (Plutarque, Artax. 19.4-5) 51 ? En fait, c'est une plaisanterie : son nom désigne un «trompeur», mais, comme aliment, il évoque le terme phonétiquement proche de "phénix $»^{52}$, quoique cette créature soit égyptienne chez Hérodote, comme aussi chez le poète comique Antiphane de Rhodes ${ }^{53}$ : sans doute estce plutôt ici le grand coq céleste oriental, « celui qui apparaît ». Ce bon mot, que nous proposons de rendre littéralement par "l'apparitrompeur ", sert à railler un homme politique contemporain ${ }^{54}$.

Pour revenir aux auteurs chrétiens, au siècle d'Eusèbe, Épiphane introduit également par le même verbe paív $\omega$ sa présentation du mythe du phénix, qu'il soit conscient ou

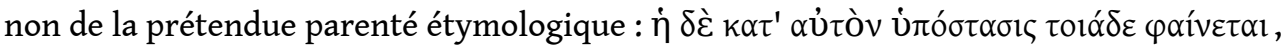
littéralement « la matière à ce sujet se présente ainsi » (ÉPIPHANE, Ancor.84). Cyrille de Jérusalem emploie lui aussi plusieurs fois des mots sur ce radical de $\varphi \alpha i ́ v \omega$ dans son

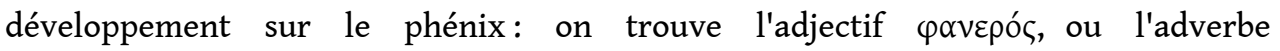
correspondant, quand il explique que l'exemple de cet oiseau "fait apparaître ", ou "rend apparente» la possibilité matérielle de la résurrection des corps. Trois occurrences se succèdent en quelques lignes, ainsi qu'un synonyme : la résurrection des

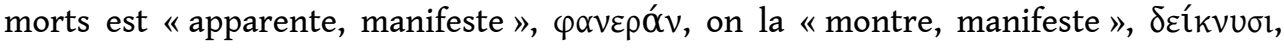

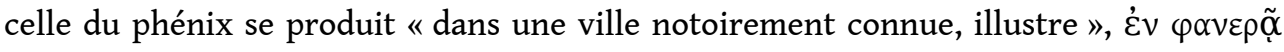

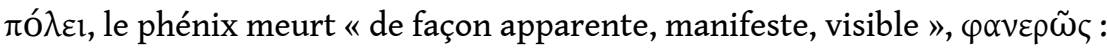

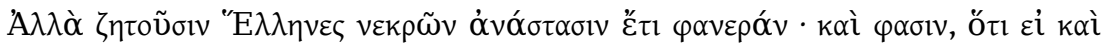

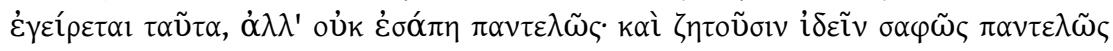

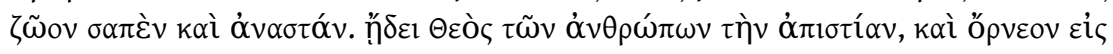

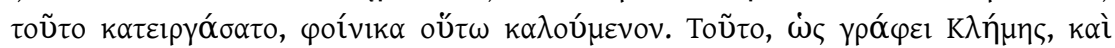

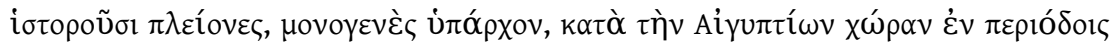

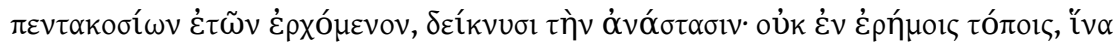

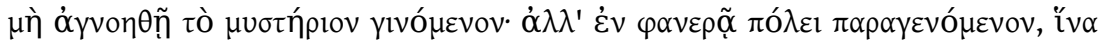

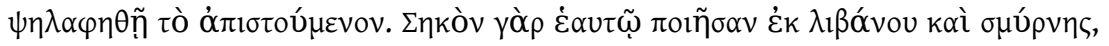

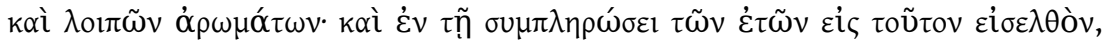

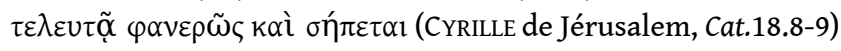

Mais les Grecs demandent une résurrection des morts encore plus manifeste, et ils disent que même si ces êtres ressuscitent, ils n'étaient cependant pas complètement décomposés; ils demandent à voir clairement un être vivant décomposé et ressuscité. Dieu connaissait l'incrédulité des hommes, et il créa un oiseau à cette fin, ainsi appelé phénix. Celui-ci, comme l'écrit clément, et comme beaucoup le racontent, unique de son espèce, se rend tous les cinq cents ans dans le pays des Égyptiens et il y manifeste la résurrection, non dans des déserts, de peur que le 
mystère qui s'accomplit reste ignoré, mais il se produit dans une ville illustre, pour que l'incroyable soit tangible. Car l'oiseau se construit un nid d'encens, de myrrhe et autres herbes aromatiques; il y entre au terme du nombre de ses années, et, de façon manifeste, il y meurt et se décompose ${ }^{55}$. grande merveille de la révélation chrétienne, la résurrection des corps, est l'illustration éclatante de ce qu'écrivait Saint Paul dans son Épittre aux Romains à propos d'un tout autre sujet :

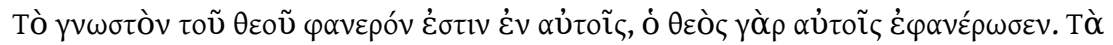

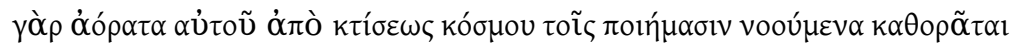
Ce que l'on peut connaître de Dieu est pour eux [les hommes] manifeste : Dieu le leur a manifesté. En effet, depuis la création du monde, ses perfections invisibles, éternelle puissance et divinité, sont visibles dans ses œuvres pour l'intelligence (PAUL, Romains 1.19-20) $)^{56}$.

\subsection{Une créature solaire syncrétique}

Doté d'une telle étymologie, ce phénix, bien que chrétien, semble partager quelques traits avec une divinité de la théogonie orphique : Phanès, « celui qui apparaît » ${ }^{57}$. Dieu ailé, comme l'est Éros, mais aussi comme l'est un oiseau; né d'un œuf, comme un oiseau ; protogène, comme l'Éros de la cosmogonie hésiodique ${ }^{58}$ et comme le benu de la cosmogonie héliopolitaine ${ }^{59}$; hermaphrodite comme le phénix selon certaines interprétations des textes ${ }^{60}$, et parfois même sortant d'un œuf enflammé ${ }^{61}$, comme le

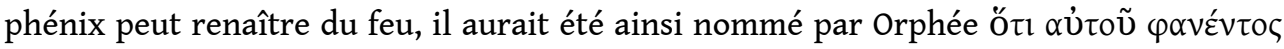

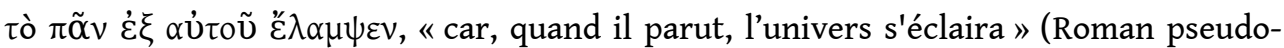
clémentin, Homélie 6.5.4) $)^{62}$ : c'est une apparition lumineuse. On représentait ce dieu dans le cercle du zodiaque (fig. 11), comme le phénix d'un aureus de l'empereur Hadrien (fig. 12), comme également l'allégorie d'Aiôn, le «temps éternel » auquel le phénix est associé sur une monnaie de l'empereur Antonin ${ }^{63}$ (fig. 13). Le nom de Phanès est donné comme l'un de ceux d'une divinité également adorée en tant que Hélios (le Soleil) et que Mithra, le dieu perse dont un des grades d'initiés s'appelle " héliodrome » : il s'agit là d'un fonds commun syncrétique de dénominations, d'attributs et de chiffres mystiques de nature solaire.

\subsection{Un coq céleste}

Mais revenons au coq héliodrome : il est assimilé au phénix dans deux autres œuvres de nature ésotérique: explicitement dans l'Apocalypse grecque $\mathrm{d} u$ Pseudo-Baruch et implicitement dans le traité magique des Cyranides.

Le premier texte est un pseudépigraphe judéo-chrétien du $2^{\mathrm{e}}$ siècle. Après l'évocation rapide d'un serpent marin infernal avatar du Léviathan, on y voit, dans le troisième ciel, là où « le soleil traverse et donne la lumière au monde » (7.1), un volatile grand " comme neuf montagnes ", nommé " phénix ». Il vole aux côtés du conducteur du char d'Hélios : c'est encore l'oiseau qui « apparaît ». À l'opposé du serpent et contrairement au monstre ailé $\mathrm{Ziz}$, il joue un rôle bénéfique, qui est longuement détaillé.

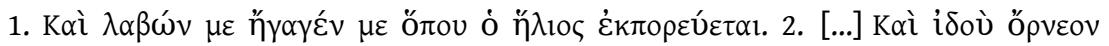

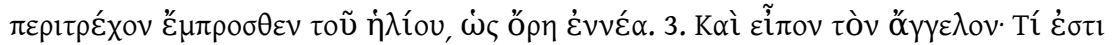

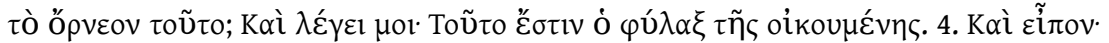

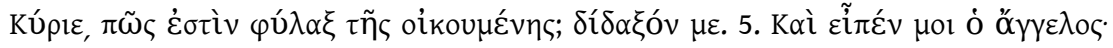




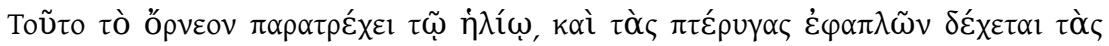

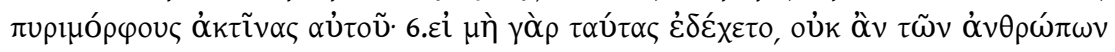

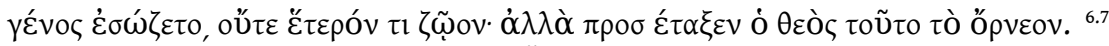

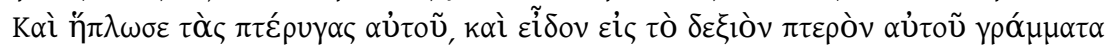

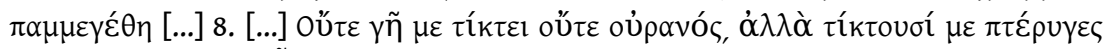

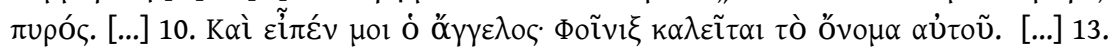

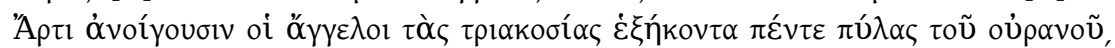

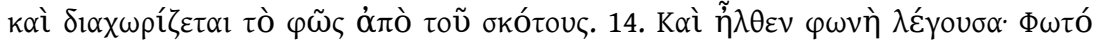

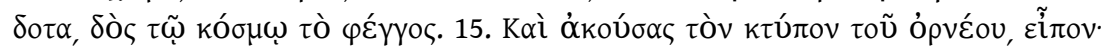

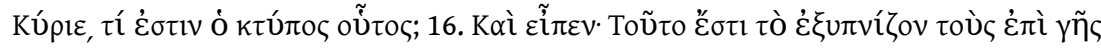

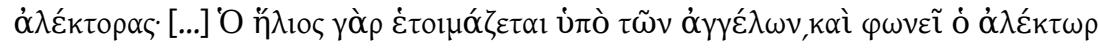
[...] (Ps. BARUCH, Apoc. 6.1-16)

Et <l'ange $>$ me conduisit à l'endroit où le soleil commence sa carrière ; [...] et voici qu'un oiseau se mit à courir en avant du soleil, et il était grand comme neuf montagnes; et je dis à l'ange : 'Quel est cet oiseau ?' Et il me dit : 'C'est le gardien de la terre habitée.' Et je dis : 'Seigneur, comment est-il le gardien de la terre habitée? Apprends-le moi.' Et l'ange me dit: 'Cet oiseau court aux côtés du soleil, et déployant ses ailes, il en reçoit les rayons enflammés; et s'il ne les interceptait point, la race des hommes ne pourrait vivre, non plus qu'aucune autre espèce animale, mais Dieu a commis l'oiseau <à cette tâche>'. Et l'oiseau étendit ses ailes et je vis sur son aile droite des lettres gigantesques [...] : 'Ni la terre ne m'engendre ni le ciel : ce sont les ailes du feu qui m'engendrent' [...]. L'ange me dit : ‘Phénix est le nom qu'il porte.' [...] 'En cet instant, les anges viennent d'ouvrir les 365 portes du ciel, et la lumière se sépare de l'obscurité'. Et il s'éleva une voix qui disait: 'Dispensateur de la lumière, dispense à l'univers la clarté'. Et ayant entendu le bruit que faisait l'oiseau, je dis : 'Seigneur, quel est ce bruit?' Et il me dit: ‘Ceci est le signal du réveil des coqs sur la terre [...]. Le soleil vient d'être apprêté par les anges ; voila pourquoi le coq chante.' [... $]^{64}$.

On ne trouve ici aucun des principaux thèmes du mythe du phénix: la mort et la renaissance cyclique. L'oiseau intercepte la brûlure du soleil : il est "gardien » de la

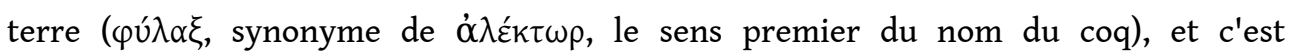
conformément au sens étymologique d'héliodrome, " coureur du soleil ", qu'il court, plutôt qu'il ne vole. Il est associé à son parcours quotidien quand s'ouvrent les 365

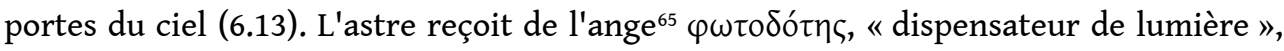
l'ordre de faire surgir la lumière, $\varphi \varepsilon ́ \gamma \gamma o \varsigma$, un synonyme poétique de $\varphi \omega ́ \varsigma$, lui-même

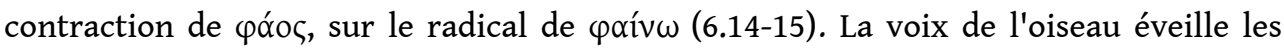
coqs terrestres dont il est le chef céleste. C'est donc l'annonce par ce coq en chef de la clarté du jour qui lui vaut l'appellation de $\varphi$ oĩvi $\xi$, ce qui est le nom de sa fonction : le phénix est encore ici l'oiseau " qui apparaît », comme l'exprime un composé du verbe

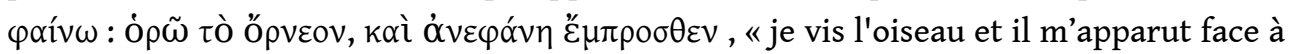
face $»(7.3)$.

Le second texte assimilant le coq héliodrome au phénix, les cyranides, est une compilation byzantine de textes attribués à Hermès Trismégiste et Harpocration d'Alexandrie, que l'on situe au IVe siècle au plus tôt ${ }^{66}$; y figure une liste d'animaux très proche de celle des Physiologus. Les livres II - IV sont des bestiaires, consacrés aux quadrupèdes, aux oiseaux et aux poissons. Si l'œuvre cite le coq ordinaire, le phénix ne s'y rencontre étonnamment pas, mais il y a un oiseau héliodrome, dont c'est l'unique attestation antique. Cette créature semble tenir la place du phénix, avec lequel elle présente des ressemblances (comme aussi avec le coq solaire), mais elle n'en a pas la nature spécifique de mourir et renaître périodiquement. Dans une courte notice, sa présentation est suivie de ses propriétés médico-magiques. ${ }^{67}$ 


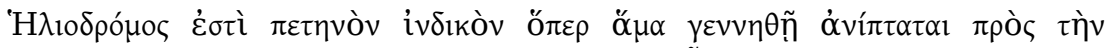

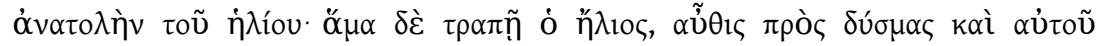

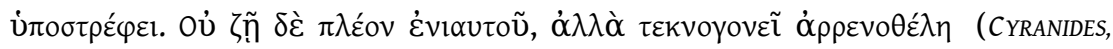
3.15)

L'héliodrome est un oiseau indien qui, aussitôt né, vole vers le soleil qui se lève, mais quand le soleil a tourné, alors il vole vers le couchant. Il ne vit pas plus d'une année, mais il enfante des petits, mâles et femelles ${ }^{68}$.

otons qu'il suit et non pas précède la course du soleil, à la façon de la fleur héliotrope qui est sa correspondante végétale symbolique, comme nous l'avons montré, car il se déplace face à lui et non dans le même sens ${ }^{69}$. Les différences avec le mythe classique du phénix sont nettes : l'héliodrome est mortel et il engendre une progéniture plurielle et

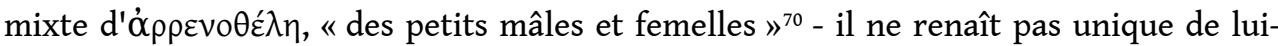
même. Mais l'héliodrome est indien comme le phénix d'une certaine tradition ${ }^{71}$ et il est lui aussi associé au chiffre 365: dans le Physiologus de Vienne, c'est le nombre des plumes du « phénix » géant; chez Baruch, c'est le nombre des portes du ciel. Ici, c'est le nombre des jours de l'année solaire, qui est à la fois la durée de vie de l'héliodrome et le temps qui lui est nécessaire pour être en âge de procréer.

31

On trouvait aussi le chiffre douze pour le nombre des sources de l'oasis d'Élim où apparaissait l'oiseau mystérieux dans la pièce d'Ézéchiel le Tragique, et dans la Bible avant lui ${ }^{72}$. Ce même chiffre - qui est aussi le nombre des rayons du nimbe de l'oiseau sur la plupart des monnaies (voir fig. 13) - est répété avec insistance sous diverses formes et à divers propos dans la longue poésie que Lactance a consacrée au phénix ${ }^{73}$. De fait, décrivant l'oiseau comme un croisement entre paon et faisan, tous deux originaires de l'Inde (LACTANCE, De aue phoen.143-144.), il lui attribue la fonction de satellite de Phébus-Apollon, comme l'est le coq céleste, et de marqueur des heures, comme l'est le coq terrestre ordinaire ${ }^{74}$. Cette assimilation avec le roi de la basse-cour se voit encore chez Claudien, auteur du second grand poème antique sur le phénix ${ }^{75}$ : dans une autre œuvre, l'éloge de Stilicon, il présente le général de Théodose en ambassade à la cour du roi de Perse tel un phénix sous des allures de $\operatorname{coq}^{76}$, avec une crête rouge ${ }^{77}$ comme un couvre-chef royal perse ${ }^{78}$; nous avons vu dans la première partie de cet article que le Physiologus du Ps. Épiphane remplace la couronne habituelle du phénix par une tiare, coiffe typiquement perse - encore un signe de l'orientalisation de l'oiseau (fig. 14 et 15). Pour le Stilicon à la fois coq et phénix de Claudien, c'est le dieu-soleil sur son char qui l'accompagne, dans une inversion des rôles par rapport à l'héliodrome de l'Apocalypse du Ps. Baruch et des Cyranides.

Les Chrétiens font d'ailleurs aussi du coq un symbole religieux ${ }^{79}$ : il a ses poètes, Ambroise et Prudence ${ }^{80}$, comme le phénix a Lactance et Claudien. Mais pas plus de coq que de phénix dans l'Ancien Testament, ni en hébreu, ni en grec: seule la Vulgate latine y introduit le $\mathrm{coq}^{81}$.

\section{Conclusion}

Pour conclure, le phénix de la description du Physiologus de Vienne n'est pas une créature très orthodoxe, car il est abondamment contaminé par le coq héliodrome bienfaisant tel que dans l'Apocalypse du Ps. Baruch, et l'est aussi un peu par l'oiseau juif malfaisant aux pattes palmées (qu'on le nomme Ziz ou autrement), tandis que l'explication étymologique nous oriente vers le dieu orphique Phanès dans un vaste syncrétisme ésotérico-religieux qui se retrouve aussi dans le recueil hermétique des 
Cyranides. Sous le nom de "phénix » dans les deux premières œuvres, et d'héliodrome, " coursier du soleil» dans la troisième, ces textes parlent en fait du coq cosmique oriental. Dans le Physiologus de Vienne, c'est dans le titre et dans la première partie (la description) que le phénix est un coq héliodrome ${ }^{82}$; ce n'est que dans le reste de la notice (la partie narrative) qu'on trouve le récit classique attendu, dépourvu cependant de certains quelques détails qui renvoient au coursier du soleil ${ }^{83}$.

\section{BIBLIOGRAPHIE}

\section{I. Éditions de textes}

ARIStophane, Comédies, vol. 3 : Les Oiseaux, traduction de Hilaire van DAELE, Paris, Les Belles Lettres, Collection des universités de France, 1928, rééd. 1967.

Claudien, CEuvres complètes, traduction de Charles-H. HÉGUIN DE GUERLE et d'Alphonse TROGNON, Paris, Panckoucke, 1830-1833, 2 vol.

CYRANIDES : Lapidaires grecs, texte et traduction de Fernand DE MÉLY, Paris, Ernest Leroux, 1902 ; Die Kyraniden, Dimitris KAIMAKIs (éd.), Meisenheim-am-Glan, Hain, 1976.

CYRILLE de Jérusalem, Catéchèses, MIGNE Jacques-Paul (éd.), Patrologia graeca,33, Paris, Garnier, 1857.

ÉLIEN : Élien, La personnalité des animaux, Livres X à XVIII, traduit et commenté par Arnaud ZUCKER, Paris, Les Belles Lettres, « La roue à livres », 2002.

ÉPIPHANE : Physiologus, MIGNE Jacques-Paul (éd.), Patrologia graeca,43, Paris, Garnier, 1864.

EusÈBE de Césarée, Préparation évangélique, traduction du Marquis Nicolas Séguier de SaintBrisson, Paris, Gaume Frères, 1846.

ÉZÉCHIEL le Tragique : L'Exagoge d'Ézéchiel le Tragique. Introduction, texte, traduction et commentaire, Pierluigi LANFRANCHI (éd.), Leiden - Boston, Brill, 2006.

HORTUS SANITATIS : Hortus sanitatis : Livre IV, Les Poissons, Catherine JACQUEMARD, Brigitte GAUVIN, Marie-Agnès LUCAS-AVENEL (éd.), avec la collaboration de Caroline FÉVRIER et Françoise LECocQ, Caen, Presses universitaires, Collection Fontes \& Paginae, 2013 (édition également en ligne sur https://www.unicaen.fr/puc/sources/depiscibus/accueil).

NonNos DE PANopolis, Dionysiaques, traduction du Comte DE MARCELlus, Paris, Firmin Didot, 1856.

PATRologie : Patrologia Graeco-Latina, Jacques-Paul MIGNE (éd.), Paris, Garnier, 1844-1866, 161 vol.

PHYSIOLOGUS SYRUS: Physiologus syrus, seu Historia animalium XXXII, Olaus Gerhardus TYCHSEN (éd.), Rostock, Stiller, 1795.

ORPHIQUES : Orphicorum fragmenta, Otto KERN (éd.), Berlin, Weidmann, 1922. 


\section{Travaux}

ALMAGOR Eran, «Ctesias and the importance of his writings revisited », Electrum, 19, 2012, p. 9-40. BEAUX Nathalie, «Le fruit et l'oiseau - Écriture de bn dans les Textes des Pyramides », Études et Travaux XXVI. 1, Institut des cultures méditerranéennes et orientales de l'Académie polonaise des sciences, Warszawa, 2013, p. 104-119.

BERNAND André, « Les lapsus d'un diplomate [Aristophane, les Acharniens, v. 82] », Métis, 6.1-2, 1991, p. 115-117.

BRISSON Luc, Le sexe incertain. Androgynie et hermaphrodisme dans l'Antiquité gréco-romaine, Paris, Les Belles Lettres, 1997.

BuSWELL Jr. Robert E., LOPEZ Jr. Donald S., The Princeton Dictionary of Buddhism, Princeton , Princeton University Press, 2013.

CôTÉ Dominique, « La figure d'Éros dans les Homélies pseudoclémentines », in Coptica - Gnostica Manichaica. Mélanges Funk, POIRIER Paul-Huber, PAINCHAUd Louis (éd.), Québec - Louvain, Presses de l'Université Laval - Peeters, 2005, p. 135-165.

Cumont Franz, « Le coq blanc des Mazdéens et les Pythagoriciens », Comptes rendus des séances de l'Académie des Inscriptions et Belles-Lettres, 86, 1942, p. 284-300.

DAumas Michèle, « Aristophane et les Perses », Revue des Études Anciennes, 87, 3-4, 1985, p. 289-305.

DUYRAT Frédérique, « Tigrane en Syrie : un prince sans images. Suivi d'un catalogue des monnaies de Tigrane le Grand dans les collections du Département des Monnaies, Médailles et Antiques de la Bibliothèque nationale de France ", Cahiers des études anciennes, 49, 2012, p. 167-209.

FeStUGIÈRE André-Jean, « Le symbole du Phénix et le mysticisme hermétique », Monuments Piot, 38, 1941, p. 147-151.

Frojmovic Eva, « A Sense of Order : Text and Image in some of the Earliest illuminated Hebrew Manuscripts from Ashkenaz », in Zu Bild und Text im jüdisch-christlichen Kontext im Mittelalter (Erfurter Schriften zur Jüdischen Geschichte, vol. 3), BusSERT Frank, LAUBENSTEIN Sarah, STÜRZEBECHER Maria (éd.), Jena - Quedlinburg, Bussert \& Stadeler, 2014, p. 48-65.

GERHARD Iohannes, Loci theologici, Jena, Steinmann, 1610-1625, Preuss Friedrich R. E. (rééd.), Schlawitz, Berlin, 1870, tome 8, Locus 26, Tractatus secundus : De mortuorum resurrectione. Gosserez Laurence, Poésie de lumière. Une lecture de Prudence, Peeters, Louvain - Paris, 2001. Guilleux Nicole, «L'étymologie de phénix : un état des lieux », in Phénix. Mythe(s) et signe(s), Fabrizio-Costa Silvia (éd.), Bern, Peter Lang, 2001, p. 9-27.

HubAux Jean et LEROY Maxime, Le mythe du phénix dans les littératures grecque et latine, Liège Paris, Droz, 1939.

KULIK Alexander, « The mysteries of Behemoth and Leviathan and the celestial bestiary of 3Baruch », Muséon, 122.3-4, 2009, p. 307-345.

LAZARIS Stavros, Le Physiologus grec, vol. I, La réécriture de l'histoire naturelle antique, Firenze, SISMEL, Edizioni del Galluzzo, 2016.

LECLERCQ-MARX Jacqueline, « Drôles d'oiseaux. Le caladre, le phénix, la sirène, le griffon et la serre dans le Physiologus, les Bestiaires et les grandes encyclopédies du XIII ${ }^{\mathrm{e}}$ siècle. Mise en 
perspective ", in Déduits d'oiseaux au Moyen Âge, ConNochiE-Bourgne Chantal (éd.), Aix-enProvence, Publications de l'université de Provence (« Senefiance » 54), 2009, p. 163-178.

LECocQ Françoise, «L’empereur romain et le phénix », in Phénix. Mythe(s) et signe(s), FABRIzIo-Costa Silvia (éd.), Bern, Peter Lang, 2001, p. 27-56.

LECOCQ Françoise, «Les sources égyptiennes du mythe du phénix », in L'Égypte à Rome, Françoise LECocQ (éd.), Cahiers MRSH-Caen, nº 41, 2e éd. augmentée 2008 (1ère éd. 2005), p. 211-266.

LECOCQ Françoise, « L'œuf du phénix. Myrrhe, encens et cannelle dans le mythe du phénix », Schedae [En ligne], 6.1 | 2009 : L'animal et le savoir, p. 73-106.

LECOCQ Françoise, « L'iconographie du phénix à Rome », Schedae [En ligne], 17.2 | 2009 : Images de l'animal dans l'Antiquité, p. 107-130.

LECOCQ Françoise, « Le roman indien du phénix ou les variations romanesques du mythe du phénix ", in Présence du roman grec et latin, Poignault Rémy, Dubel Sandrine (éd.), Caesarodunum XL-XLI bis, Clermont-Ferrand, Centre Piganiol, 2011, p. 405-429.

LECocQ Françoise, «Le phénix dans l'œuvre de Claudien : la fin d'un mythe », in Claudien. Mythe, histoire et science, GARAMBOIS-VASQUEZ Florence (éd.), Saint-Étienne, Presses universitaires, 2011, p. 113-157.

LECOCQ Françoise, "Kinnamômon ornéon ou phénix ? L'oiseau, la viande et la cannelle », in Prédateurs dans tous leurs états. Evolution, biodiversité, interactions, mythes, symboles, Jean- BRUGAL Philip, GARDEISEN Armelle, ZuCKER Arnaud (éd.), Antibes, APDCA, 2011, p. 409-420.

LECOCQ Françoise, « 'Le sexe incertain’ du phénix : de la zoologie à la théologie », in Le phénix et son Autre. Poétique d'un mythe, Laurence GosSEREZ (éd.), Rennes, Presses Universitaires, 2013, p. $189-210$.

LECOCQ Françoise, «L'oiseau Phénix de Lactance : uariatio et postérité (de Claudien au poème anglosaxon The Phoenix) », in La uariatio :l'aventure d'un principe d'écriture, de l'Antiquité au XXIe siècle, Hélène ViaL (éd.), Paris, Garnier, 2014, p. 185-201.

LECOCQ Françoise, « Y a-t-il un phénix dans la Bible ? À propos de Job 29.18, de Tertullien, De resurrectione carnis 13, et d'Ambroise, De excessu fratris 2, 59 », Kentron, 30, 2014, p. 55-81.

LECOCQ Françoise, « Un bilan de la recherche contemporaine sur le mythe du phénix », Roda da fortuna [En ligne] 1 | 2015, p. 257-273.

LECOCQ Françoise, "Inventing the Phoenix : a Myth in the Making through Words and Images ", in Animals in Greek and Roman Religion and Myth, Johnston Patricia A., MASTRocinque Attilio, Papaioannou Sofia (éd.), Cambridge, Cambridge Scholars Publishing, 2016, p. 449-478 ${ }^{84}$.

Mermier Guy René, « The Phoenix : Its Nature and Its Place in the Tradition of the Physiologus ", in Beasts and Birds of the Middle Ages. The Bestiary and its Legacy, CLARK Willene B., MCMUNN Meradith T. (éd.), Philadelphia, University of Pennsylvania Press, 1989, p. 69-87.

NAGY Árpád M., «Le phénix et l'oiseau-benu sur les gemmes magiques », in Phénix. Mythe(s) et signe(s), FABRizio-Costa Silvia (éd.), Bern, Peter Lang, 2001, p. 57-84.

NAGY Árpád M., « Magical Gems and Classical Archaeology », in 'Gems of Heaven' : Recent Research on Engraved Gemstones in Late Antiquity, c. AD 200-600, ENTwISTLE Chris, ADAMS Noël (éd.), London, The British Museum, 2011 p. 75-81.

OlBRICH Konstantin, « Athanasius, die Kaiser und der Anbruch einer neuen Ära : Propaganda und Münzprägung um 343 n. Chr. », Klio 86.2, 2004, p. 415-441. 
PETERS John P., « The Cock », Journal of the American Oriental Society, 33, 1913, p. 363-396.

PICARD Jean-Claude, Le Continent Apocryphe. Essai sur les littératures apocryphes juive et chrétienne, Brepols, Steenbrugis - Turnhout, 1999 («Le phénix, la tour et les hybrides : syncrétisme et comparatisme ", p. 107-122).

PINTUS Giovanna Maria, « Storia di un simbolo : il gallo », Sandalion, 8-9,1985-1986, p. 243-286.

QUET Marie-Henriette, «L'aureus au zodiaque d'Hadrien, première image de l'éternité cyclique dans l'idéologie et l'imaginaire temporel romains ", Revue numismatique, 160, 2004, p. 119-154. QUIRKE Stephen, The Cult of Ra. Sun-Worship in Ancient Egypt, London, Thames \& Hudson, 2001.

ROESSLI Jean-Michel, « La cosmo-théogonie orphique du roman pseudo-clémentin : Note sur ses sources et son utilisation dans les Homélies et les Reconnaissances », Les Études Classiques, 76, 1, 2008, p. 83-94.

RonzitTI Rosa, « Il gallo nell'Avesta, nel Veda e in Leopardi », Rivista italiana di linguistica e di dialettologia, 14, 2012, p. 29-64.

SBORDONE Francesco, « La fenice nel culto di Helios », Rivista indo-greco-italica, 19, 1935, p. 1-46.

Somville Pierre, « Jeux de mots et sens du sacré dans la religion grecque », Kernos, 2, 1989, p. 199-211.

TuPLIN Christopher, Persian Responses: Political and Cultural Interaction With(in) the Achaemenid Empire, Swansea, Classical Press of Wales, 2007.

TURDEANU Émile, « Notule I. Une curiosité de l'Hénoch slave : les phénix du sixième ciel », Revue des études slaves, 47, 1968, p. 53-54.

VAN DEN BROEK, Roelof, The Myth of the Phoenix according to Classical and Early Christian Traditions, Leiden, Brill, 1972.

VERMASEREN Maarteen J., Corpus Inscriptionum et Monumentorum Religionis Mithriacae, Den Haag, Martinus Nijhoff, 1956-1960.

WACHOLDER Ben Zion, Bowman Steven, «Ezechielus the Dramatist and Ezekiel the Prophet : Is the Mysterious $\zeta \tilde{\omega}$ ov in the 'E $\xi \alpha \gamma \omega \gamma \eta$ a Phoenix ? », Harvard Theological Review, 78, 3/7, 1985,

p. 253-277.

WAZANA Nili, « Anzu and Ziz: Great Mythical Birds in Ancient Near Eastern, Biblical, and Rabbinic Traditions », Journal of the Ancient Near Eastern Society, 31, 2008, p. 111-135.

ZUCKER Arnaud, Physiologos, le bestiaire des bestiaires, Grenoble, Jérôme Millon, 2004 (phénix p. 83-88).

ZURETTI Carlo Oresto, « Per la critica del Physiologus greco », Studi italiani di Filologia classica, 5, 1897, p. 113-219.

\section{NOTES}

1. Rien de tel encore pour le premier phénix iconographique romain, celui de la taverne d'Euxinus à Pompéi, dont la sacralité se manifeste par un toupet et une barbiche, comme pour les serpents divins ; seules ses couleurs ocre jaune et rouge évoquent sans doute l'éclat solaire. Cette ville montre aussi le seul benu italien dans son temple d'Isis, voir F. LECOCQ, "Les premières peintures du phénix, à Pompéi », Actes du XXIXe colloque de l'Association française pour la peinture 
murale antique (Louvres, 18-19 novembre 2017), J. BOISLÈVE, A. DARDENAY, F. MONIER (éd.), Bordeaux, Ausonius (Collection Pictor), à paraître en novembre 2018.

2. PLINE L'ANCIEN, HN 10.5, voir F. LECOCQ, «L'empereur romain et le phénix », in Phénix. Mythe(s) et signe(s), S. FABRIzIO-CoSTA (éd.), Bern, Peter Lang, 2001, p. 27-56.

3. Voir F. LECOCQ, « Deux faces du phénix impérial : Trajan et Hadrien sur l'aureus de 117/118 », in Mémoires de Trajan, mémoires d'Hadrien, S. BENOIST, A. GAUTIER, C. HOËT-VAN CAUWENBERGHE, R. Poignault (éd.), Lille, Septentrion, à paraître en 2019.

4. Sauf peut-être sur les gemmes magiques gréco-égyptiennes, qui sont d'époque romaine tardive (2e-4e s.), et dont l'iconographie peut avoir été influencée par celle des monnaies impériales au phénix, voir Á. M. NAGY, «Le phénix et l'oiseau-benu sur les gemmes magiques ", in Phénix. Mythe(s) et signe(s), S. FABrizio-costA (éd.), Bern, Peter Lang, 2001, p. 57-84, et « Magical Gems and Classical Archaeology ", in 'Gems of Heaven' : Recent Research on Engraved Gemstones in Late Antiquity, c. AD 200-600, C. ENTWISTLE, N. ADAMS (éd.), London, The British Museum, 2011, p. 75-81.

5. Voir G. R. Mermier, "The Phoenix : Its Nature and Its Place in the Tradition of the Physiologus ", in Beasts and Birds of the Middle Ages. The Bestiary and its Legacy, W. B. CLARK, M. T. MCMunN (éd.), Philadelphia, University of Pennsylvania Press, 1989, p. 69-87.

6. Voir par exemple le cas de Pline l'Ancien dans F. LECOCQ, «Kinnamômon ornéon ou phénix ? L'oiseau, la viande et la cannelle ", in Prédateurs dans tous leurs états. Evolution, biodiversité, interactions, mythes, symboles, J.-Ph. BRUGAL, A. GARDEISEN, A. ZuCKER (éd.), Antibes, APDCA, 2011, p. 409-420.

7. Ce travail de recherche a été présenté oralement sous forme de "notule» en 2013 à l'université de Caen - Normandie, lors de l'hommage rendu à notre collègue Olivier Desbordes à l'occasion de son départ en retraite.

8. ÉPIPHANE, Physiologus, 11, J.-P. MIGNE (ed.), Patrologia graeca,43, col. 525-528, traduction latine de l'édition de G. PONCE DE LEON, Antwerpen, Plantin, 1588 ( $1^{\mathrm{e}}$ édition Rome, 1587).

9. Le texte grec dit d'ailleurs non pas « couronne », mais « tiare », mot dont la translittération en latin est attestée chez les auteurs du 1er siècle : c'est un des couvre-chefs royaux perses, ce qui nous rapproche du portrait très oriental de l'oiseau brossé par Claudien dans son poème Phoenix (CM 27). Voir la seconde partie de cet article.

10. Voir le fac-simile en ligne sur http://spcoll.library.uvic.ca/Digit/physiologum/facsimile/ facsimile_img.htm, consulté le 14.05.2018.

11. D. B ADKE, en date de 2014, sur http://spcoll.library.uvic.ca/Digit/physiologum/animal/ phoenix.htm, consulté le 14.05.2018. Il y a une double glose dans sa traduction puisque la couronne devient une crête ou huppe, bref des plumes - comme effectivement dans d'autres textes, mais certainement pas ici.

12. Seul un mot grec rapproche la cheville d'un feu métaphorique à propos de la douleur

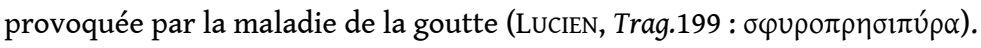

13. Par exemple CLAUDIEN, CM 27.21 : (...) Tyrio pinguntur crura ueneno, « (...) ses jambes sont teintes de la pourpre de Tyr », traduction de C.-H. HÉGUIN DE GUERLE, Paris, Panckoucke, 1833, tome 2.

14. Une seule exception : l'indication de pieds palmés dans le Physiologus de Vienne, texte que nous examinons dans la seconde partie de cet article ; la forme de palme n'a rien à voir avec les « malléoles ».

15. Notre traduction. Sur la présence du phénix au paradis, voir F. LECOCQ, «The Flight of the Phoenix towards Paradise in Literature and Iconography", in Animal Kingdom of Heaven. Anthropozoological Aspects of the Late Antique World, I. ScHAAF (éd.), Berlin, De Gruyter, 2018.

16. Physiologus syrus, seu Historia animalium XXXII, Rostock, Stiller, 1795.

17. Notre traduction; voir J. HUBAUX, M. LEROY, Le Mythe du phénix dans les littératures grecque et latine, Liège - Paris, Droz, 1939, p. 158, R. VAN DEN BROEK, The Myth of the Phoenix according to Classical and Early Christian Traditions, Leiden, Brill, 1972, p. 204. 
18. Loci theologici, Steinmann, Jena, 1610-1625, F. R. E. Preuss (rééd.), Schlawitz, Berlin, 1870, tome 8, Locus 26, Tractatus secundus: De mortuorum resurrectione, p. 435 (notre traduction).

19. F. SBORDONE, « La fenice nel culto di Helios », Rivista indo-greco-italica, 19, 1935, p. 1-46, p. 19-20.

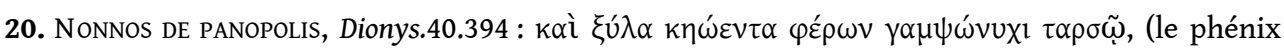
qui) «apporte dans ses serres recourbées des rameaux odoriférants » (traduction du Comte de Marcellus, Paris, Didot, 1856).

21. Voir le dossier iconographique de l'ouvrage du savant néerlandais (VAN DEN BROEK, The Myth of the Phoenix, 1972 : 423-464) et notre étude (F. LECOCQ, "L'iconographie du phénix à Rome ", Schedae [En ligne], 17.2 | 2009: Images de l'animal dans l'Antiquité, p. 107-130). Les fils de Constantin frappent, en alternance avec le phénix au globe, un phénix sur un entassement pyramidal de ce qui semble des rochers, dont l'interprétation n'est pas assurée (c'est le sujet de notre communication « Les réinterprétations textuelles et symboliques des attributs iconographiques du phénix, de l'Égypte ancienne à nos jours ", colloque Images sources de Textes, Textes sources d'Images, 18-20 juin 2018, Université de Saint-Étienne, Y. Dureau, S. Coin-Longeray dir.).

22. Voir VAN DEN BROEK, The Myth of the Phoenix, $1972: 234$, n. 4.

23. Voir C. O. ZURETTI, «Per la critica del Physiologus greco ", Studi italiani di Filologia classica, 5, 1897, p. 113-219, p. 153, texte 2. Le même manuscrit comporte aussi vers la fin une seconde notice sur cet oiseau, sans description (HUBAUX, LeRoy, Le mythe du phénix, 1939 : 154, texte 3). Le ms F 68 est en ligne sur http://www.internetculturale.it: la phrase en question est à la p. 158v de la pagination en rouge $=p .170 \mathrm{v}$ de la pagination en noir ; consulté le 09.04.2018).

24. Voir F. LECOCQ, "Un bilan de la recherche contemporaine sur le mythe du phénix ", Roda da fortuna [En ligne] 1| 2015, p. 257-273, et « Inventing the Phoenix : a Myth in the Making through Words and Images", in Animals in Greek and Roman Religion and Myth, P. A. JoHnston, A. Mastrocinque, S. PAPAIOANnou (éd.), Cambridge, Cambridge Scholars Publishing, 2016, p. 449-478 (consulter de préférence la version en ligne, exempte des fautes de l'éditeur).

25. Traduction de HUBAUX, LEROY, Le mythe du phénix, 1939 : XXXV-XXXVI.

26. Voir VAN DEN BROEK, The Myth of the Phoenix, $1972: 296$.

27. Hérodote n'avait pas signalé l'homonymie avec phoinix, " palmier », si fructueuse par la suite dans l'enrichissement et l'imagerie du mythe, non seulement chez les auteurs grecs, mais même et surtout chez les auteurs latins (pour qui « palmier » se dit palma) grâce à leur bilinguisme : par exemple Ovide, Mét. 15.396, Pline L'ANCIEN, HN13.42, LACTANCE, De aue phoen.69-70). La brève interpretatio proposée par le Physiologus du Ps. Épiphane ne fait apparaître l'homonyme végétal de l'oiseau que dans une citation partielle du Psaume 91.13: Iustus ut phoenix florebit, ut cedrus in

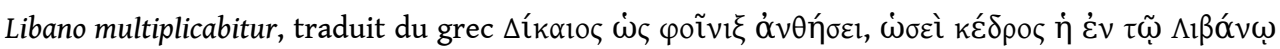
$\pi \lambda \eta \theta u v \theta \eta \dot{\sigma \varepsilon \tau \alpha l, ~ « L e ~ j u s t e ~ g r a n d i r a ~ c o m m e ~ u n ~ p a l m i e r, ~ i l ~ p o u s s e r a ~ c o m m e ~ u n ~ c e ̀ d r e ~ d u ~ L i b a n ~ » ; ~}$ cette citation est reprise de Tertullien qui joue sur l'ambiguïté du mot grec à la double signification, oiseau et arbre (TERTULLIEN, De res. mort. 13.2) ; voir F. LECOCQ, « Y a-t-il un phénix dans la Bible ? À propos de Job 29.18, de Tertullien, De resurrectione carnis 13, et d'Ambroise, De excessu fratris 2, 59 ", Kentron, 30, 2014, p. 55-81.

28. Voir K. OlbRich, « Athanasius, die Kaiser und der Anbruch einer neuen Ära : Propaganda und Münzprägung um 343 n. Chr. », Klio, 86.2, 2004, p. 415-441.

29. Nous pensons ici plutôt à un autre cas d'erreur textuelle, dont la source serait une notice sur Taprobane, c'est à dire Ceylan, la patrie de la cannelle indienne, l'aromate préférentiel du phénix depuis sa confusion avec l'oiseau cannelle (voir n. 6). Cette notice parle de ses animaux et de ses palmeraies - mais pas du phénix : « l'île dans la grande mer, celle qu'ils appellent Taprobane,

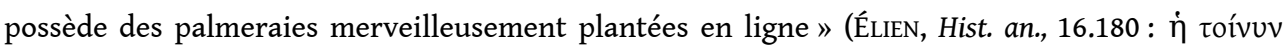

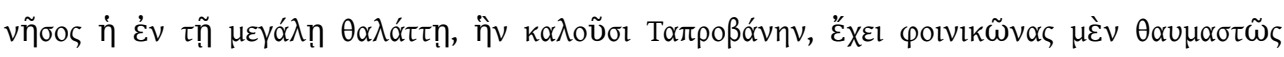

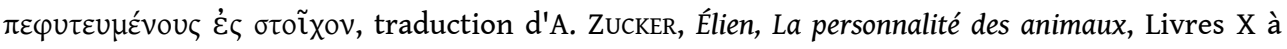
XVIII, Paris, Les Belles Lettres, « La roue à livres », 2002). Les trois mots que nous avons soulignés 
se retrouvent en effet dans la phrase du Physiologus de Vienne, et « Athanase » y serait donc un

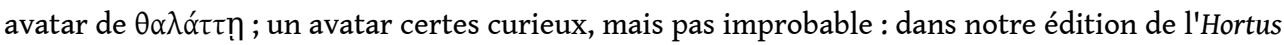
sanitatis, œuvre qui est une compilation de même type que le Physiologus, nous avons trouvé l'auteur Trebius Niger (attesté dans PLINE L'ANCIEN, HN 9.80-81), transformé en un imaginaire poisson noir, le trebius niger (Hortus sanitatis : Livre IV, Les Poissons, C. JACQUEMARD, B. GAUVIN, M.-A. LuCAS-AVENel (éd.), avec la collaboration de C. FÉVRIER et F. LeCocQ, Caen, Presses universitaires, Collection Fontes \& Paginae, 2013, chap. 4.93). Notre proposition d'une mélecture conduisant à l'apparition d'Athanase dans le texte du Physiologus ne remet cependant pas en cause l'indice de datation de l'œuvre que constitue la mention de ce nom (voir S. Lazaris, Le Physiologus grec, vol. I, La réécriture de l'histoire naturelle antique, Firenze, SISMEL, Edizioni del Galluzzo, 2016, p. 22).

30. Voir N. WAZANA, «Anzu and Ziz: Great Mythical Birds in Ancient Near Eastern, Biblical, and Rabbinic Traditions ", Journal of the Ancient Near Eastern Society, 31, p. 111-135, p. 113-116.

31. Voir R. RonzITTI, « Il gallo nell'Avesta, nel Veda e in Leopardi », Rivista italiana di linguistica e di dialettologia, 14, 2012, p. 29-6, p. 30-34.

32. Voir R. E. BusweLl Jr., D. S. LOPEZ Jr., The Princeton Dictionary of Buddhism, Princeton, Princeton University Press, 2013, s. V., p. 249-250.

33. Voir WAZANA, « Anzu and Ziz », $2008: 119-124$.

34. Voir E. Frojmovic, "A Sense of Order: Text and Image in some of the Earliest illuminated Hebrew Manuscripts from Ashkenaz », in Zu Bild und Text im jüdisch-christlichen Kontext im Mittelalter (Erfurter Schriften zur Jüdischen Geschichte, vol.3), F. BUSSERT, S. LAUBENSTEIN, M. STÜRZEBECHER (éd.), Jena - Quedlinburg, Bussert \& Stadeler, 2014, p. 48-65, p. 62-64 et fig. 3.

35. Tractate Berakhot, fol.57b, British Library, vers 1280. Bar Iokni est généralement identifié comme une autruche (dont les pattes ont deux doigts non palmés).

36. Dans ces deux œuvres, l'arbre tire son nom de l'oiseau ; Isidore écrit que tous deux partagent une longue durée de vie.

37. LACTANCE, De aue phoen.65-66: Dirigit in Syriam celeres longaeua uolatus, / Phoenicis nomen cui dedit ipsa Venus, "Vif en dépit des ans, il s'envole en Syrie / Qui reçut de l'oiseau son nom de Phénicie " (traduction de Hubaux, Leroy, Le mythe du phénix, 1939: XVII, aux vers numérotés 45-46). C'est aussi la Phénicie qu'il faut reconnaître dans les diverses appellations de "Syrie » (LACTANCE, De aue phoen. 65), et même d'« Assyrie » (OvidE, Mét. 15.393), ou encore de "Liban » dans le Physiologus (avec, là, un jeu de mots de plus entre le terme géographique latin et le nom grec de l'encens : $\lambda i ́ \beta \alpha v o s$, et / ou une référence biblique au pays des cèdres, voir F. LECoce Françoise, «L'œuf du phénix. Myrrhe, encens et cannelle dans le mythe du phénix », Schedae [En ligne], 6.1 | 2009 : L'animal et le savoir, p. 73-106, et LECOCQ, « Inventing the Phoenix », $2016: 456$ et 470-471.

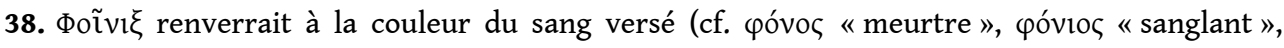

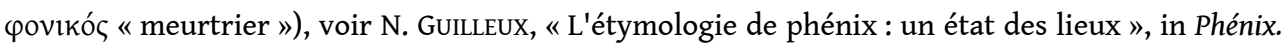
Mythe(s) et signe(s), S. FABRIzIO-CostA (éd.), Bern, Peter Lang, p. 9-27, p. 22 ; la couleur du plumage de l'oiseau correspond aussi à celle des fruits mûrs du palmier-dattier. Pour le nom égyptien de l'oiseau benu, «celui qui surgit », voir N. BEAUX, «Le fruit et l'oiseau - Écriture de bn dans les Textes des Pyramides", Études et Travaux XXVI.1, Institut des cultures méditerranéennes et orientales de l'Académie polonaise des sciences, Warszawa, 2013, p. 104-119, p. 114-117.

39. Texte et traduction de HUBAUX, LEROY, Le mythe du phénix, 1939 : XXXVI.

40. Voir A. ZUCKER, Physiologos, le bestiaire des bestiaires, Grenoble, Jérôme Millon, 2004, p. 83 et 86-87.

41. Voir F. CUmont, « Le coq blanc des Mazdéens et les Pythagoriciens », Comptes rendus des séances de l'Académie des Inscriptions et Belles-Lettres, 86, 1942, p. 284-300, p. 290-291 et 297-299.

42. Fragment 16, v. 246-247, traduction de P. L ANFRANCHI, L'Exagoge d'Ézéchiel le Tragique. Introduction, texte, traduction et commentaire, Leiden - Boston, Brill, 2006, p. 273. 
43. On trouve la même précision dans la traduction grecque de la Septante pour Job 29. 18, faite

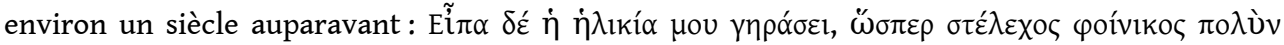

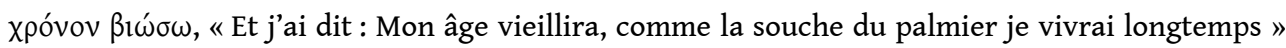
(notre traduction), voir LECOCQ, « Y a-t-il un phénix dans la Bible?», 2014 : 58-59.

44. Traduction de N. Séguier de Saint-Brisson, Paris, Gaume Frères, 1846.

45. Citation tirée d'ÉzÉCHIEL Le TRAGIQUe, Ex., fragment 17, v. 258, 263 et 265 (notre traduction d'après LANFRANCHI, L'Exagoge, 2006 : 283-284).

46. Pour l'identification de l'oiseau avec un aigle, voir B. Z. WACHOLDER, S. BOWMAN, «Ezechielus

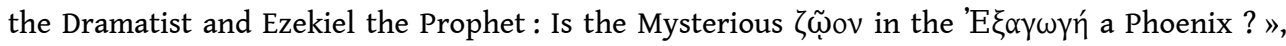
Harvard Theological Review, 78, 3/7, 1985, p 253-277.

47. Ps. Eustathe, Comm. in Hex., PG 18, 729C-732A.

48. Citation tirée d' ÉZÉCHIEL LE TRAgiQUE, Ex., fragment 17, v. 260-262, notre traduction d'après

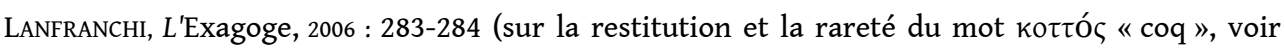
p. 287). On entend littéralement un « cocorico » dans les nombreuses allitérations en $\kappa-$.

49. Voir F. LECOCQ, "'Le sexe incertain' du phénix: de la zoologie à la théologie », Laurence Gosserez (éd.), Le phénix et son Autre. Poétique d'un mythe, Rennes, Presses Universitaires, 2013, p. 189-210, p. 193-195.

50. On trouve $\varphi \varepsilon ́ v \alpha \xi$, avec une déformation volontaire (ARISTOPHANE, Acharn. 89), phoenix en latin, phinix dans la langue tardive (GRÉGOIRE DE TOURS, Du cours des étoiles, 12, qui offre un résumé du poème de Lactance), et fenix sur le sceau du diacre Sicirius, et encore fins en syriaque (transcription phonétique) sur la mosaïque funéraire d'Edessa (voir VAN DEN BROEK, The Myth of the Phoenix, 1972 : pl. XXX et XIII pour ces deux derniers documents).

51. Voir E. AlMAGOR, «Ctesias and the importance of his writings revisited », Electrum 19, 2012, p. 9-40, p. 19 et n. 76.

52. Cette référence n'a pas été repérée par HUBAUX, LEROY, Le mythe du phénix, 1939, ni par VAN DEN BRoEK, The Myth of the Phoenix, 1972.

53. Les compatriotes, dans ATHÉNÉE, Deipn. 14.655b.

54. Mais il faut bien sûr une traduction qui puisse correspondre à un nom de plat: on a proposé par exemple le «faisan farceur» (A. BERNAND, "Les lapsus d'un diplomate [Aristophane, les Acharniens, v. 82] », Métis, 6.1-2, 1991, p. 115-117). Le jeu de mots se prolonge sur le vers suivant et la pièce fait également plus loin référence à Phénix le précepteur d'Achille (ARISTOPHANE, Acharn. 421-422).

55. Notre traduction. Il est difficile de trouver en français des mots de même racine pour toutes les occurrences du radical de $\varphi \alpha v \varepsilon \rho o ́ c$, comme nous avons essayé de le faire. Le problème se posait déjà en latin, où les expressions que nous avons relevées sont rendues par: conspicuam mortuorum resurrectionem, resurrectionem ostendit, in urbe conspicua, moritur palam (J.-P. MIGNE (éd.), Patrologia graeca,33, col. 1026, traduction latine reprise d'Antoine Augustin Touttée, Paris, 1720). Nous n'avons à ce jour relevé qu'un seul texte, non littéraire, où est employé le verbe latin apparere au sujet du phénix, mais il n'est pas certain que le jeu de mots bilingue y soit conscient vu la nature épigraphique du document: dans les Fasti Vindobonenses priores, on lit pour la prétendue exhibition de l'oiseau à Rome en 48 sous Claude à l'occasion du $800^{\mathrm{e}}$ anniversaire de la ville, Vitellio II et Publicola his cons. phenix apparuit primum, « Sous les consuls Vitellius (son second consulat) et Publicola, le phénix apparut pour la première fois » (notre traduction), voir vAN DEN BROEK, The Myth of the Phoenix, $1972: 116$, n. 3).

56. Texte cité et traduit par S. LAZARIS, Le Physiologus grec, 2016, p. 3 et 114.

57. Là encore, ce jeu de mots est propre à la langue grecque et nécessite une explication en latin. Rufin d'Aquilée glose ainsi : Phaneta $[m]$ (...) ab apparendo, «Phanès (...) de 'apparaître' » (Rufin, Recogn. 10.17). 
58. Voir D. CôTÉ, «La figure d'Éros dans les Homélies pseudoclémentines », in Coptica - Gnostica Manichaica. Mélanges Funk, P.-H. PoIRIER, L. PAInchaud (éd.), Québec - Louvain, Presses de l'Université Laval - Peeters, 2005, p. 135-165.

59. Voir S. QUIRKE, The Cult of Ra. Sun-Worship in Ancient Egypt, London, Thames \& Hudson, 2001, p. 27-30, et F. LECOCQ, «Les sources égyptiennes du mythe du phénix », in L'Égypte à Rome, Cahiers MRSH-Caen, nº 41, F. LECocQ (éd.), 2e éd. augmentée 2008 ( $1^{\mathrm{e}}$ éd. 2005), p. 211-266, p. 212-220.

60. À partir de la restitution hypothétique du vers $163 \mathrm{du}$ poème de Lactance avalisée par HubAUX, LeROY, Le mythe du phénix, 1939: XX (avec une traduction qui ne correspond pas au texte latin retenu), eux-mêmes suivis par VAN DEN BROEK, The Myth of the Phoenix, 1972 : 364-365 et 374-375, et par L. BRISSON, Le sexe incertain. Androgynie et hermaphrodisme dans l'Antiquité grécoromaine, Paris, Les Belles Lettres, 1997, p. 24-37. Contra, voir F. LECocQ, «'Le sexe incertain' du phénix ", $2013:$ 199-201.

61. Sur le relief de Modène, Phanès sort d'un œuf environné de flammes et entouré par les douze signes du zodiaque (CIMRM 695, dans M. J. VeRMASEREN, Corpus Inscriptionum et Monumentorum Religionis Mithriacae, Den Haag, Martinus Nijhoff, 1956-1960).

62. Orphicorum Fragmenta, 56, O. KERN (éd.), Berlin, Weidmann, 1922, p. 134, notre traduction. Voir J.-M. ROESSLI, « La cosmo-théogonie orphique du roman pseudo-clémentin : Note sur ses sources et son utilisation dans les Homélies et les Reconnaissances", Les Études Classiques, 76, 1, 2008, p. 83-94, p. 90.

63. Voir F. LECOCQ, « Deux faces du phénix impérial », à paraître en 2019.

64. Traduction de HubAuX, LeRoy, Le mythe du phénix, 1939: XXIX-XXXI. L'Hénoch slave, autre pseudépigraphe juif, montre des créatures composites géantes tirant le char du soleil ; elles sont appelées « chalkydri » et «phénix » (2Enoch, 12. 1, voir WAZANA, « Anzu and Ziz », 2008 : 125-129, et A. KulIK, "The Mysteries of Behemoth and Leviathan and the Celestial Bestiary of 3Baruch », Muséon, 122.3-4, 2009, p. 307-345, p. 339). Plus loin, les «phénix » du sixième ciel ne sont qu'une mélecture de l'hébreu ophanim « roues » (2Enoch, 19. 3, voir E. TURDEANU, « Notule I. Une curiosité de l'Hénoch slave : les phénix du sixième ciel », Revue des études slaves, 47, 1968, p. 53-54).

65. Mais de l'oiseau dans la version slavonique (voir KULIK, «The Mysteries of Behemoth », 2009 : 310).

66. C'est à dire un dieu des sciences occultes et un adepte d'Harpocrate, divinité grécoégyptienne d'un culte à mystères. L'ouvrage est prétendument traduit du syriaque en grec.

67. Voir F. LECOCQ, «Deux oiseaux solaires en un: le coq, le phénix et l'héliodrome ", in Inter litteras \& scientias. Mélanges C. Jacquemard, B. GAUVIN et M.-A. LUCAS-AVEnel (éd.), Caen, Presses universitaires, 2019.

68. Texte grec des Lapidaires grecs de F. DE MÉLY, Paris, E. Leroux, 1902, traduction de HubAUX, LEROY, Le mythe du phénix, 1939: 7-8 (le texte grec cité par ces derniers omet involontairement quelques mots).

69. Voir n. 67.

70. Un terme rare, où l'on a voulu voir des hermaphrodites, voir n. 60. La graphie correcte de

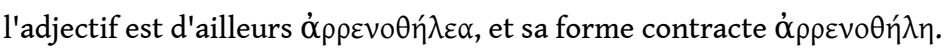

71. Voir F. LECOCQ, «Le roman indien du phénix ou les variations romanesques du mythe du phénix ", in Présence du roman grec et latin, R. PoignaUlt, S. Dubel (éd.), Caesarodunum XL-XLI bis, Clermont-Ferrand, Centre Piganiol, 2011, p. 405-429.

72. Ce chiffre a là un autre symbolisme, en référence au nombre des tribus d'Israël, voir P. LANFRANCHI, L'Exagoge d'Ézéchiel le Tragique : 278-279.

73. De aue phoen. 8, 28, $37=73,38=74$ (HUBAUX, LEROY, Le mythe du phénix, 1939 : XI-XIII).

74. De aue phoen.3-34: compagnon du soleil, 44-46 (=80-82): chant matinal, et 55-56 (=35-36): indication des heures (HUBAUX, LEROY, Le mythe du phénix, 1939 : XI-XIII). 
75. Carmina Minora, 27 : Phoenix, voir F. LECocQ, «Le phénix dans l'œuvre de Claudien : la fin d'un mythe ", in Claudien. Mythe, histoire et science, F. GARAMBoIs-VASQuEz (éd.), Saint-Étienne, Presses universitaires, 2011, p. 113-157, p. 117-123. Pour une comparaison avec le poème de Lactance, voir LECOCQ, «L'oiseau Phénix de Lactance : uariatio et postérité (de Claudien au poème anglo-saxon The Phoenix)", in La uariatio : l'aventure d'un principe d'écriture, de l'Antiquité au XXIe siècle, H. VIAL (éd.), Paris, Garnier, 2014, p. 185-201, p. 192-197.

76. Cons. Stil. 2.416-419 et 422-423. Voir LECOCQ, «Le phénix dans l'œuvre de Claudien », 2011 : 117-124.

77. Claudien, CM27.18-19: rutilo uertice (...) cristatus apex, « un appendice en forme de crête à pointe(s) rouge(s)» (traduction littérale). La périphrase cristatus ales (ou auis) est fréquente en poésie pour désigner le coq (par exemple OVIDE, Mét.11.597 et F.1.455; MARTIAL, Ép.9.68.3 et 14.223.2). On donne à ce dernier une crête d'un «rouge phénicien" (THÉocRITE, Id.22.72:

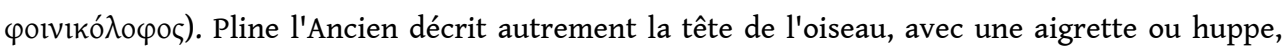
mais son expression cristis fauces pourrait désigner les caroncules du coq, puisque l'oiseau porte littéralement des « crêtes » à la gorge (PLINE L'ANCIEN, HN10.3).

78. Claudien suit en cela la description du coq d'A RISTOPHANE, Ois.481-486: "D'abord je vous citerai le coq, qui était tyran et commandait aux Perses, avant tous, les Darieos et Mégabazos, si bien qu'on l'appelle oiseau de Perse, en raison de cette antique souveraineté. - C'est donc pour cela que maintenant encore, pareil au Grand Roi, il fait de grands pas, et sur sa tête, seul de tous les oiseaux porte la tiare droite » (traduction d'H. vAN DAELE, Paris, Les Belles Lettres, Collection des universités de France, 1928, rééd. 1967). Sur la tiare comparée à une crête de coq, voir C. TuPLIN, Persian Responses : Political and Cultural Interaction With(in) the Achaemenid Empire, Swansea, Classical Press of Wales, 2007, p. 68-73, et M. DAumAs, «Aristophane et les Perses ", Revue des Études Anciennes, 87, 3-4, 1985, p. 289-305, p. 292-293. Telle qu'on la voit sur un tétragramme du 1er s. avant notre ère, la coiffe de Tigrane II, roi d'Arménie d'origine perse, présente de fait une couronne dentelée ornée de deux aigles et d'une étoile (le soleil ?), avec des pans de tissu rabattus sur les oreilles, très semblables aux crête et caroncules du coq ; si le tissu était rouge, la similitude deviendrait frappante (voir F. DUYRAT, « Tigrane en Syrie : un prince sans images. Suivi d'un catalogue des monnaies de Tigrane le Grand dans les collections du Département des Monnaies, Médailles et Antiques de la Bibliothèque nationale de France ", Cahier des études anciennes, 49, 2012, p. 167-209).

79. Voir G. M. PiNTUS, «Storia di un simbolo : il gallo", Sandalion, 8-9, 1985-1986, p. 243-286, p. 261-267.

80. Ambroise, Hymnus Aeterne rerum conditor, strophes 5 et 6, Prudence, Cath. I., Ad galli cantum, qui est un long hymne de 100 vers (voir L. Gosserez, Poésie de lumière. Une lecture de Prudence, Peeters, Louvain - Paris, 2001, p. 67-76).

81. Voir J. P. PETERS, "The Cock », Journal of the American Oriental Society, 33, 1913, p. 363-396, p. 366-370).

82. Contrairement à ce que pensent HuBAUX, LeROY, Le mythe du phénix, 1939 : 9-13, suivis par A.- J. Festugière, «Le symbole du Phénix et le mysticisme hermétique », Monuments Piot, 38, 1941, p. 147-151, p. 147-148.

83. Dans l'Apocalypse grecque de Baruch, c'est un griffon qui tient ce rôle de coursier du soleil, voir VAN DEN BROEK, The Myth of the Phoenix, 1972: 272, 292 et 303, KULIK, « The mysteries of Behemoth », 2009 : 335, et plus généralement, J.-C. PICARD, Le Continent Apocryphe. Essai sur les littératures apocryphes juive et chrétienne, Brepols, Steenbrugge-Turnhout, 1999, Chap. « Le phénix, la tour et les hybrides : syncrétisme et comparatisme », p. 107-122. Sur le griffon, voir J. LECLERCQMARX, « Drôles d'oiseaux. Le caladre, le phénix, la sirène, le griffon et la serre dans le Physiologus, les Bestiaires et les grandes encyclopédies du XIII ${ }^{\mathrm{e}}$ siècle. Mise en perspective ", in Déduits d'oiseaux 
au Moyen Âge, C. Connochie-bourgne (éd.), Aix-en-Provence, Publications de l'université de Provence (« Senefiance » 54), 2009, p. 163-178.

84. Cet article a malheureusement été publié avec plusieurs fautes dues aux éditeurs; voir de préférence la version originale en ligne sur notre page d'academia.edu ou de researchgate.edu.

\section{RÉSUMÉS}

Le christianisme a très tôt adopté le mythique phénix comme preuve naturelle de la résurrection des corps. Le bestiaire du Physiologus classe ainsi l'oiseau en bonne place. Traduite en plusieurs langues, à diverses époques, cette œuvre populaire s'est accrue de variantes qui renvoient à des sources précises, mais qui peuvent aussi être des inventions ou même des erreurs. Dans le Physiologus byzantin du pseudo-Épiphane, l'une de ces variantes s'explique par la mélecture du

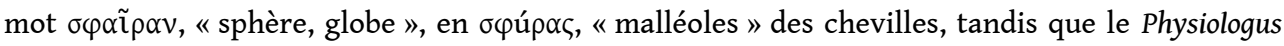
grec de Vienne propose une explication étymologique du nom de l'oiseau comme «celui qui apparait », du verbe paív $\omega$; elle repose sur une assimilation du phénix au légendaire coq

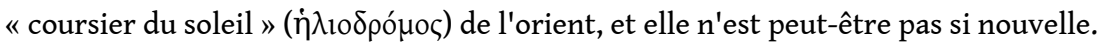

Christianity very early adopted the mythical phoenix as natural proof of the resurrection of the bodies. So, the Physiologus lists the bird in a good position. Translated into several languages, at various times, this popular work expanded with variants that either come from definite sources, or appear as being sheer inventions, or even errors. In the Byzantine Physiologus of the pseudoEpiphanius, one of these variants is explainable by the misreading of the word $\sigma \varphi \alpha \tilde{i} \rho \alpha v$, «sphere,

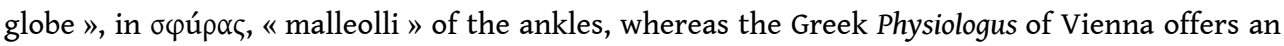
etymological explanation of the name of the bird as "the one that appears ", from the verb poív $\omega$; it is based on an assimilation of the phoenix to the legendary western cock « runner of

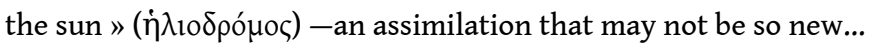

\section{INDEX}

Keywords : Phoenix, Greek Physiologus, Misreading, Etymology, Heavenly Rooster

Mots-clés : Phénix, Physiologus grec, mélecture, étymologie, coq céleste

\section{AUTEUR}

\section{FRANÇOISE LECOCQ}

Françoise Lecocq est Maître de Conférences en Latin, Université de Caen - Normandie, au Centre Michel de Boüard - CRAHAM (

UMR 6273). Spécialiste du monde romain, ses travaux portent surtout sur les mythes, en particulier le mythe d'Europe et le mythe du phénix qu'elle étudie sous de multiples facettes (sa place dans la Bible, son sexe, l'importance des parfums et aromates, etc.). 